\title{
Comunicación entre la enfermera y el familiar: una relación entre seres humanos honesta, directa y real*
}

\author{
Communication between the Nurse and the Family Member: An Honest, Direct, and Authentic \\ Relationship between Human Beings \\ A comunicação entre o enfermeiro e o familiar: uma relação honesta, direta e real entre o ser humano
}

Luz Patricia Díaz Heredia ${ }^{\text {a }}$

Universidad Nacional de Colombia, Colombia

lpdiazh@unal.edu.co

ORCID: https://orcid.org/0000-0002-7167-282X

Gersson Andrés Ballesteros Pinzón

Universidad Nacional de Colombia, Colombia

ORCID: https://orcid.org/0000-0002-7879-8249
DOI: https://doi.org/10.11144/Javeriana.ie23.cefr

Recibido: 20 Febrero 2020

Aceptado: 17 Octubre 2020

Publicado: 15 Septiembre 2021

\section{Resumen:}

Introducción: la estancia en la unidad de cuidados intensivos (UCI) genera en el paciente y sus familias sentimientos de falta de control, incertidumbre y ansiedad que se encuentran relacionados con falta de información y comunicación tanto sobre el proceso de salud-enfermedad vivido como sobre el tratamiento. Objetivo: describir las características de la comunicación en un etnos determinado entre la enfermera y el familiar del paciente hospitalizado. Método: estudio cualitativo con enfoque microetnográfico que utilizó para el análisis la propuesta de Bardin. Los participantes fueron 12 familiares de pacientes hospitalizados en la UCI adultos de las ciudades de Bucaramanga y Floridablanca (Colombia) durante el 2016. Se realizó entrevista semiestructurada con cinco preguntas sobre la comunicación con el profesional de enfermería. Resultados: en su mayoría, los participantes fueron mujeres, universitarias e hijas procedentes de ciudades diferentes del lugar de la entrevista. Surgió un tema principal: la comunicación: una relación entre seres humanos honesta, directa y real, conformado por cuatro categorías: la información como eje central de la comunicación en UCI; la comunicación que no se expresa con palabras, aún más importante en la relación; la comunicación no fluye si no hay tiempo, disposición y condiciones para entablar la relación; y la comunicación genera sentimientos positivos y satisfacción. Conclusión: la comunicación como proceso social enmarcado en la UCI como etnos particular demanda de la enfermera profesional cualidades como amabilidad, cordialidad, empatía, reconocimiento y lenguaje entendible, al igual que la realimentación y disposición para entablar y mantener este proceso vital para las relaciones humanas.

Palabras clave: comunicación, cuidados críticos, enfermería, familia, DeCS.

\section{Abstract:}

Introduction: The intensive care unit (ICU) stay generates feelings of lack of control, uncertainty, and anxiety in the patient and their families related to a lack of information and communication about the health-disease process experienced and the treatment. Objective: to describe the characteristics of communication in the ethnos between the nurse and the family member of the hospitalized patient. Method: a qualitative study with a micro ethnographic approach that used Bardin's proposal for the analysis. The participants were 12 relatives of patients hospitalized in the adult ICU in Bucaramanga and Floridablanca (Colombia) during 2016. A semi-structured interview was conducted with five questions about communication with the nurse practitioner. Results: most of the participants were women, university students, and daughters from cities other than the place of the interview. The main theme that emerges was communication: an honest, direct, and authentic relationship between human beings, made up of four categories: information as the central axis of communication in ICU; communication that is not expressed in words, which is even more important in the relationship; communication does not flow if there is no time, disposition, and conditions to establish the relationship; and communication generates positive feelings and satisfaction. Conclusion: communication as a social process framed in the ICU as a particular ethnos demand from the nurse practitioners' qualities such as kindness, cordiality, empathy, recognition, and understandable language, as well as feedback and willingness to establish and maintain this vital process for human relationships. Keywords: communication, critical care, nursing, family. DeCS

Keywords: communication, critical care, nursing, family, DeCS.

\section{Notas de autor}

\footnotetext{
${ }^{a}$ Autora de correspondencia. Correos electrónicos: lpdiazh@unal.edu.co y lpdiazh@gmail.com
} 
Resumo:

Introdução: a permanência na Unidade de Terapia Intensiva (UTI) gera no paciente e suas famílias, sentimento de falta de controle, incerteza e ansiedade relacionados com falta de informações e comunicação tanto sobre o processo saúde-doença vivido quanto sobre o tratamento. Objetivo: descrever as características da comunicação em um determinado etnos entre enfermeiro e familiar do paciente internado. Método: estudo qualitativo com abordagem microetnográfica que utilizou a proposta de Bardin para a análise. Os participantes foram 12 familiares de pacientes internados na UTI adulto das cidades de Bucaramanga e Floridablanca (Colômbia) durante o 2016. Foi realizada entrevista semiestruturada com cinco questões sobre a comunicação com o profissional de enfermagem. Resultados: a maioria dos participantes foram mulheres, universitárias e filhas provindas de outras cidades que não o local da entrevista. Emergiu um tema central: a comunicação: relação honesta, direta e real entre os seres humanos, composta por quatro categorias: a informação como eixo central da comunicação em UTI; comunicação que não se expressa em palavras, ainda mais importante na relação; a comunicação não flui se não houver tempo, disposição e condições para estabelecer o relacionamento; e a comunicação gera sentimentos positivos e satisfação. Conclusão: a comunicação como processo social enquadrado na UTI como etnos particular exige do profissional enfermeiro qualidades como gentileza, cordialidade, empatia, reconhecimento e linguagem compreensível, bem como feedback e disposição para estabelecer e manter esse processo vital para as relações humanas.

Keywords: communication, critical care, nursing, family, DeCS.

Palavras chave: comunicação, cuidados críticos, enfermagem, família, DeCS

\section{Introducción}

El ingreso de un paciente en una unidad de cuidados intensivos (UCI) somete a los miembros de la familia a una situación difícil, angustiante y con una gran carga de incertidumbre (1). Por su complejidad, tanto los pacientes como sus familias presentan alteraciones emocionales como ansiedad, estrés, depresión o el denominado síndrome de cuidados intensivos (2). Además, los pacientes y las familias suelen desarrollar sentimientos de falta de control en sí mismos y de soledad, siendo las principales necesidades manifestadas por ellos las relacionadas con la información, la comunicación y la seguridad $(3,4,5)$.

Sumado a lo anterior, dentro de los aspectos que limitan la calidad en la estancia de los pacientes y sus familiares se encuentra la falta de participación de las enfermeras en los flujos de información. Sobre este particular, es común que se desista de dicha labor informativa ya que se trata de una competencia exclusiva del personal médico o porque las normas institucionales no lo facilitan (6). Así mismo, los desequilibrios de poder dentro de los equipos multidisciplinares que laboran en las UCI provocan asimetrías en su seno, generando dificultades para el manejo de información por parte de la enfermera $(7,8)$.

La investigación en relación con la familia en la UCI se ha centrado principalmente en establecer sus necesidades, dentro de las cuales está la información (9). Sin embargo, no se ha indagado sobre el reconocimiento del proceso comunicativo y la influencia del etnos presente en la UCI. La literatura consultada coincide en señalar que la necesidad de información mediada por la comunicación es una de las necesidades prioritarias (8). No poder comunicarse con el equipo de salud puede generar una serie de cambios tanto físicos como emocionales que impactan de una forma u otra en la calidad de vida de los miembros (10).

Por lo anterior, reconocer la experiencia comunicativa en un contexto social específico entre la enfermera y los familiares, expresada por ellos en el entorno de las UCI de adultos (UCIa), se convierte en el foco de la indagación de esta investigación. En este sentido, el presente documento resuelve la pregunta acerca de cómo es la comunicación entre la enfermera profesional y el familiar de la persona hospitalizada en un etnos como la unidad de cuidados intensivos. Así mismo, nos planteamos el objetivo de describir desde una perspectiva microetnográfica la comunicación entre el profesional de enfermería y el familiar de la persona hospitalizada en la UCI.

\section{Método}

Este artículo reporta parte de los resultados derivados de la tesis de Maestría en Enfermería denominada Comunicación entre la enfermera y el familiar del paciente hospitalizado en la unidad de cuidados intensivos 
(11). Se trabaja desde un enfoque microetnográfico, siguiendo las orientaciones de Akinson y Hammesley citado por Cotán (12), que se centra en la observación e interpretación del fenómeno en una sola institución social y en una o varias situaciones sociales.

En este caso, el fenómeno social es la comunicación entre la enfermera y la familia en un contexto social dado: las unidades de cuidado intensivo general polivalente de adultos ubicados en Floridablanca y Bucaramanga (Colombia). Para el análisis se utilizó el modelo propuesto por Bardin (13).

La información se obtuvo mediante una entrevista semiestructurada compuesta por cinco preguntas. La selección de los participantes se hizo de manera intencional, el muestreo fue teórico (se buscó la mayor diversidad posible de participantes para permitir más riqueza en la información) $(12,13)$ y se continuó con la recolección hasta alcanzar la saturación de los datos (hasta que no se presentaron datos nuevos en la constitución de las categorías).

La entrevista se realizó posterior a unas semanas de inmersión en campo (14) y se complementó con las notas. La observación participante se desarrolló en algunos casos antes y en otros después de las entrevistas hechas en diferentes momentos, en los cuales se realizaban los encuentros comunicativos de los participantes, y la inmersión en campo permitió, luego de un tiempo, pasar desapercibidos al interior de la unidad.

El contacto inicial fue por medio de las enfermeras profesionales del servicio y después de la entrevista, la cual fue llevada a cabo por el investigador principal, quien no hacía parte del grupo de trabajo de la institución, la grabó y luego la transcribió. Algunas de las preguntas fueron: “¿cómo describe la comunicación que ha tenido con las enfermeras profesionales del servicio?” "¿de qué manera se ha sentido durante la comunicación establecida?". Las entrevistas duraron entre 10 y 25 minutos, se realizaron antes o después de terminada la hora de visita, y finalizaron cuando los familiares expresaron no tener nada más que decir; fueron grabadas en condiciones de privacidad (15).

Respecto a los criterios de rigor, el estudio cumplió con la credibilidad (al soportar el análisis en lo referido por los familiares), la transferibilidad (al describir el contexto en el que se desarrolló la investigación), la neutralidad u objetividad (dada por la independencia de los análisis desarrollados por los dos investigadores que trabajaron en el estudio y al conjugar los datos obtenidos de la entrevista con la observación participante) y la relevancia (se obtuvo la descripción de la comunicación en un ambiente especializado, con alta carga emocional y distanciamiento físico de los participantes en el acto social de comunicación) (16). De la misma manera, el desarrollo de este estudio tuvo en cuenta la normatividad de ética en investigación propuesta en Colombia mediante la Resolución 008430 de 1993, cuyo artículo 6 establece los criterios para la investigación en seres humanos; además, se tuvo en cuenta la Declaración de Helsinki (17). Así mismo, el estudio contó con la aprobación del Comité de Ética de la Facultad de Enfermería de la Universidad Nacional de Colombia (UGI 323-2013) y solicitó el consentimiento informado de los participantes, al igual que la preservación de los principios éticos de la investigación en salud (18). El análisis de la información lo efectuó el investigador principal y un investigador con mayor experiencia, y se complementó con las notas de campo, según el esquema propuesto por Bardin (13). Se tuvieron en cuenta cuatro instancias para el análisis: la determinación del objeto o tema de análisis, las reglas de codificación, el establecimiento de las categorías y su descripción (18).

\section{Resultados}

Las características sociodemográficas de los participantes del estudio se muestran en la tabla 1. Los participantes estuvieron entre los 32 y 75 años. 
TABLA 1.

\begin{tabular}{llc}
\hline & Características & $\begin{array}{c}\text { Frecuencia } \\
\mathbf{N}=\mathbf{1 2}\end{array}$ \\
\hline \multirow{2}{*}{ Género } & Hombre & 4 \\
\hline \multirow{4}{*}{ Escolaridad } & Mujer & 8 \\
\cline { 2 - 3 } & Profesional & 8 \\
\cline { 2 - 3 } & Bachiller & 1 \\
\cline { 2 - 3 } & Primaria completa & 1 \\
\cline { 2 - 3 } Parentesco & Primaria incompleta & 2 \\
\hline \multirow{3}{*}{} & Hijo(a) & 8 \\
\cline { 2 - 3 } & Esposo(a) & 1 \\
\cline { 2 - 3 } & Tío(a) & 3 \\
\hline
\end{tabular}

Características sociodemográficas de los participantes Fuente: elaboración propia

Al efectuar el análisis de las entrevistas realizadas a los participantes del estudio, surgió un gran tema: la comunicación: una relación entre seres humanos honesta, directa y real, conformada por cuatro subtemas: 1) la información como eje central de la comunicación en UCI; 2) la comunicación que no se expresa con palabras, aún más importante en la relación; 3) la comunicación no fluye si no hay tiempo, disposición y condiciones para entablar la relación, y 4) cuando la comunicación genera sentimientos positivos y satisfacción (Fig. 1).

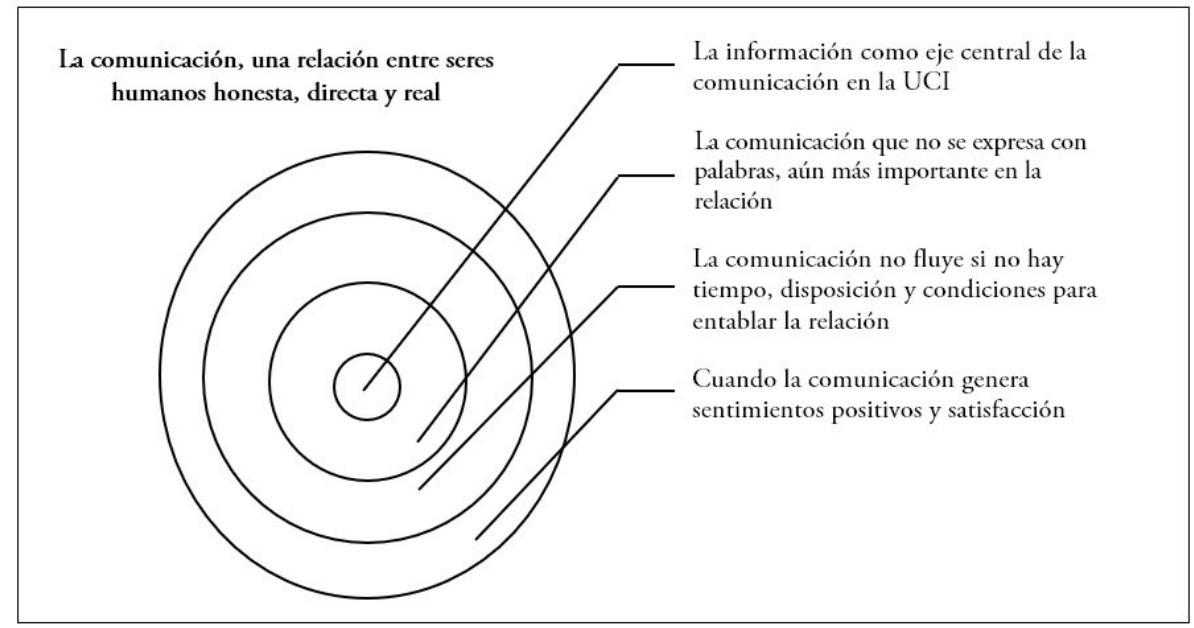

FIGURA 1.

Temas emergentes en el estudio

Fuente: elaboración propia

\section{La comunicación: una relación entre seres humanos honesta, directa y real}

La comunicación como proceso social desarrollado en el entorno de UCI está enmarcada por las vivencias, las creencias y los aspectos culturales de los participantes, y en especial por la cultura de las UCI, en donde se dificultan física y emocionalmente los encuentros comunicativos. No obstante, para el familiar, la comunicación en UCI se describe como esa relación intersubjetiva, receptiva y amigable que se puede establecer con la enfermera o el enfermero, y está relacionada principalmente con la atención brindada a su ser amado, por lo que el contexto, a pesar de ser adverso para este acto social, puede llegar a ser enriquecedor y tranquilizante.

En la relación intersubjetiva que se establece entre la diada se generan procesos dinámicos, recíprocos y de retroalimentación que se traducen en sentimientos de conformidad, satisfacción, tranquilidad y disminución 
del temor, los cuales se dan al momento de interactuar con la enfermera o el enfermero cuando estos se encuentran en el momento de visita:

Me parece que ha sido fluida... las enfermeras son más receptivas que los médicos, así como le responden a uno también se dejan preguntar cosas, así sean las preguntas que para ustedes son demasiados normales, para uno no, sí, entonces uno acá viene [...], ella me dice: "tal líquido, esto es esto, le vamos a quitar esto"; esto le genera a uno satisfacción y tranquilidad ya que le aclaran dudas relacionadas con el estado de salud de mi papá y eso es muy importante para mí [...]. (E7p2l8-15)[1]

Al mismo tiempo, la familia demanda del profesional de enfermería una comunicación basada en el respeto, el apoyo de la red primaria y secundaria, la cordialidad, la calidez, la fluidez, la honestidad y la realidad de lo observable. Así mismo, espera que se le brinde información de una forma segura, sin mentiras, franca y con un lenguaje entendible para el interlocutor, como cuando se llama a la persona por su nombre.

De esta forma, se aprecia el acto comunicativo como humano, sustentado en la interacción social generada entre los sujetos, la cual está centrada en el interés por el otro: "Porque han sido asequibles, siempre que me ha tocado iniciar una comunicación, han estado cordiales, amables y de buena manera para responder a mis preguntas y esto me tranquiliza porque me hablan del paciente [...]” (E3p2l27-29).

\section{La información como eje central de la comunicación en UCI}

La información es considerada el eje central de la comunicación en UCI, ya que tiene como propósito principal establecer la relación intersubjetiva entre el familiar y la enfermera. A su vez, el encuentro original permite fundar un vínculo emocional entre la diada, que puede trascender las dificultades o barreras físicas de reconocimiento del personal y locativas que se puedan presentar durante el acto comunicativo (11).

La información que el familiar demanda del personal de enfermería está relacionada principalmente con cómo se encuentra el paciente hospitalizado en su diario vivir dentro de la UCI. Algunos de ellos, por ejemplo, solo requieren actualización sobre los cambios en la situación clínica o patológica; en otras ocasiones, hay una doble intención en la necesidad de información con el fin de saber si las necesidades del paciente están siendo cubiertas por el personal de salud:

Pues básicamente necesito saber sobre el estado en que se encuentra mi mami en el día, cómo ha evolucionado, si ha tenido alguna complicación, si de pronto necesita algo... que necesita que le traigamos algo por ejemplo pañales, que la crema, que nosotras preguntamos de todo, que si la bañaron, si echaron las goticas en los ojos, que si... no sé, cualquier necesidad que ella tenga para nosotras estar pendiente [...]. (E12p414-8)

Así mismo, el familiar desea que el acto comunicativo se desarrolle en un lenguaje entendible, sincero, cordial, humano, y que haya coherencia entre lo que se dice y lo que se ve en la persona enferma. Lo anterior genera un ambiente propicio para que se dé la relación de ayuda, generando un marco ideal para que la comunicación sea efectiva y eficaz:

De esta visita, en particular no pues ha sido cordial, respetuosa, dan información que uno necesita, le explican cosas que uno muchas veces valora y las entendí muy fácilmente, siente uno que mi familiar les importa y yo le importo [...]. (E5p2l23-26)

\section{La comunicación que no se expresa con palabras, aún más importante en la relación}

La relación intersubjetiva que se establece con el familiar depende de cómo el profesional de enfermería inicie el acto comunicativo. Para ello resulta clave el lenguaje no verbal, entendido como todos los comportamientos o formas de actuar del profesional de enfermería sin emitir palabras. Así, se emplean recursos como gestos, movimientos de manos, ojos, expresión facial, posturas, movimientos corporales, entre otros. Es importante 
recalcar que la comunicación verbal va muy de la mano de la no verbal y ambas engloban el ideal de comunicación que esperan los familiares en el contexto UCI:

Llegó (la enfermera) con la cabeza muy en alto y con la voz alta, esa actitud siempre lo pone a uno a la defensiva [...] la actitud demasiado altiva, los hombros, la cara, los gestos de los ojos, esto como que lo predispone a uno para iniciar la comunicación con ellas [...]. (E1p5110-14)

\section{La comunicación no fluye si no hay tiempo, disposición y condiciones para entablar la relación}

La comunicación en el entorno de UCI puede presentar ciertas barreras para mantener o finalizar la interacción entre las partes, asunto que muestra de manera evidente cómo la cultura de separación y desinformación está presente en algunos de los encuentros de enfermeras y familiares. Estas dificultades están principalmente asociadas a barreras físicas o locativas, ausencia de un espacio cómodo donde se pueda brindar la información o falta de tiempo (11):

En esta UCI en cuanto a la comunicación es lo que te decía, no hay un momento específico donde se le dé la entrega de la información valiosa al familiar del paciente, sino es un momento que hay que perseguirlo, buscarlo y necesitarlo [...]. (E3p5124-27)

De la misma forma, el familiar expresa dificultades al establecer la interacción cuando el profesional de enfermería evade el acto comunicativo; en este sentido, no existe un distintivo que permita su reconocimiento o cuándo estos son agresivos o altivos:

Hay momentos en los que uno llega, este es el caso de mi familiar, es mi papá, está uno ahí, llegan quizás auxiliares o no sé si enfermeras, hay momentos en los que hay algunas de las personas que saludan, como otros que ni siquiera el saludo, parecieran que estuvieran bravos con uno, entonces uno como que se previene de preguntarles [...]. (E2p2115-18)

Negado todo, la información... la gente muy cortante, como de malgenio a toda hora... uno decía una cosa y lo hacían sentir "usted tan bruto preguntando estas bobadas", para ellos eran muy lógicas, para uno no, lo hacían a uno sentir mal por preguntar [...]. (E7p511-5)

Es importante recalcar que la familia reconoce como una de las principales causas de la imposibilidad de establecer una relación intersubjetiva con el profesional de enfermería la no identificación, la ausencia de saludo o la no presentación dentro de la UCI. Ellos expresan que no existe nada característico que los diferencie del resto del equipo de salud, por eso manifiestan sentimientos de inconformidad, porque al no identificarlos se sienten poco apoyados, se previenen de iniciar y mantener el diálogo o se corta el acto comunicativo:

Cuando uno ve a otros que se acercan y que a veces no le saludan a uno están vestidos todos también igual, o algo así, como que uno dice: "Ah... tal vez es pues el mal llamado jefe de enfermeros o enfermeras" y por eso como que no... poco hay esa comunicación e interacción con ellos y sí me gustaría hablar con las jefes [...]. (E2p3111-15)

Entonces espera uno que no sencillamente la enfermera se presente y de una vez diga: "mira listo, tú eres el familiar del paciente tal" y de una vez dan el informe y ya se acaba la comunicación por así decirlo... A veces siento que no le intereso a las enfermeras $[\ldots] .(\mathrm{E} 2 \mathrm{p} 815-12)$

\section{Cuando la comunicación genera sentimientos positivos y satisfacción}

El producto final de la relación intersubjetiva establecida entre el profesional de enfermería y el familiar puede generar sentimientos positivos como la esperanza, alegría, conformidad y satisfacción, y le permite al familiar expresar gratitud, puesto que su necesidad manifiesta de información está siendo cubierta por el profesional de enfermería y porque cumple con sus expectativas: 
O sea, porque uno se tranquiliza más con la información que ella me da. Sí... sí por la información, porque uno se tranquiliza más cuando le hablan sobre la paciente y que se lo dicen a uno de buena manera, de buena forma [...]. (E8p314-7)

Porque han sido asequibles, siempre que me ha tocado iniciar una comunicación, han estado cordiales, amables y de buena manera para responder a mis preguntas, y yo cuando vuelvo y veo a las jefes pues me acerco a ellas porque sé que me van a aclarar dudas o responder todas mis preguntas [...]. (E3p2l27-29)

\section{Discusión}

La comunicación es una actividad inherente a los seres humanos y se desarrolla en el ámbito de las relaciones entre sujetos en un espacio de configuración social (19). Para este estudio, el profesional de enfermería, al establecer el acto comunicativo honesto, directo y real con el familiar, reconoce el verdadero propósito de la interacción, buscando cumplir con las expectativas y así poder generar un lazo afectivo y de confianza. Esto permite que poco a poco se genere una relación intersubjetiva que trasforme el paradigma netamente informativo y la cultura de aislamiento, desinformación y poder que se desarrolla al interior de las UCI, de modo que transcienda hacia un cuidado relevante y auténtico. Estos aspectos también se encontraron en otros estudios con abordaje cualitativo, en los que se considera la relación interpersonal un propósito conocido y aceptado por ambas partes $(19,20,21,22,23)$.

Construir estrategias que tengan como objetivo una atención centrada en la persona, como la utilización de un lenguaje accesible, la escucha atenta, una sonrisa o una expresión de confianza, una mirada que demuestre tranquilidad, un tacto afectivo que proporcione apoyo y consuelo o una palabra que anime y eleve a los pacientes, son elementos encontrados en otros estudios $(24,25)$.

La comunicación no puede ser un acto de trasmisión de mensajes en un solo sentido. Esto resulta todavía más importante en las unidades de cuidado intensivo, en donde los familiares se encuentran ávidos de información (8) frente a la condición del paciente y sus necesidades como sujeto.

En este estudio, la comunicación no verbal se constituyó en un aspecto importante en la relación comunicativa. En otro estudio, en un contexto similar, se considera que los componentes verbales y no verbales que se dan en las relaciones interpersonales tienen lugar en la práctica de enfermería y están sujetos a una serie de condicionantes personales y ambientales que pueden influir para que la relación enfermera-paciente produzca los beneficios en ambos sentidos (26).

El fallo en la comunicación puede estar relacionado con la ausencia de saludo, y la falta de presentación y de identificación del personal de enfermería. Los familiares consideran que estos tres temas nombrados no les permiten establecer una comunicación eficaz. En este mismo sentido, en otro estudio cualitativo los familiares describieron la percepción que tenía el familiar del trato digno, dentro de lo cual se encontraba el saludo y la presentación del personal de enfermería ante la familia y el paciente (27).

Una de las barreras más importantes que impiden que el mensaje trasmitido sea entendido es la falta de retroalimentación. Lo anterior tiene relación con lo expresado por Campo y Cotrina (28), quienes describen que los profesionales de enfermería no dirigen la mirada al paciente o familia para no interactuar con ellos, lo cual genera una despersonalización del cuidado enfermero.

En el presente estudio fue posible identificar que cuando el profesional de enfermería evade, huye, no tiene tiempo o habilidad para la comunicación con los familiares, ellos se sienten insatisfechos, poco apoyados o aislados. Así mismo, se encontró que el profesional de enfermería reconoce que en ocasiones evita atender a la familia porque no se siente con los conocimientos y la preparación suficiente para abordar sus necesidades emocionales y tampoco dispone de estrategias de protección ante el estrés que ello le genera (28).

Es evidente que todas las estrategias que el profesional de enfermería pueda utilizar en el día a día en el desarrollo de su labor asistencial, que impliquen un contacto más estrecho y cercano con el familiar, redundan en beneficios para las dos partes; por ejemplo, en el familiar disminuyen síntomas psicológicos, se sienten 
apoyados y mejora la satisfacción, y en el profesional de enfermería permiten mejorar la comunicación, facilitan la ejecución de actividades asistenciales por la toma rápida de decisiones y ayudan al cuidado.

\section{Conclusiones}

En este estudio se pudo concluir que la comunicación en la UCI entre el familiar de la persona hospitalizada y la enfermera genera una relación entre seres humanos honesta, directa y real, concebida como fundamento de las interacciones sociales y determinada por ellas, la cual, cuando se desarrolla con las características descritas, genera confianza, tranquilidad y bienestar en la familia.

Como fenómeno permeado por la cultura de la UCI es posible identificar que reproduce el aislamiento, el uso de un lenguaje técnico y las relaciones de poder que marcan en muchos casos los encuentros comunicativos entre la enfermera y el familiar, rompiendo de manera importante la posibilidad de establecer relaciones terapéuticas y de cuidado que son importantes en la práctica diaria en dicho contexto.

\section{Financiamiento}

Esta investigación no recibió aportes financieros para su realización

\section{Conflicto de intereses}

Los autores declaran no tener conflicto de intereses

\section{Limitaciones}

Las limitaciones del estudio están relacionadas con la imposibilidad de realizar las observaciones en todos los turnos

\section{Referencias}

1. Santiago de Castro E. Experiencia de estar hospitalizado en una unidad de cuidado intensivo coronario de Barranquilla. Av. enferm. 2016;33(3):381-90. http://dx.doi.org/10.15446/av.enferm.v33n3.41841

2. Padilla-Fortunatti C, Rojas-Silva N, Arechabala-Mantuliz MC. Análisis de la diferencia entre importancia y satisfacción de las necesidades de los familiares de pacientes críticos. Med Intensiva. 2019;43(4):217-24. https: //doi.org/10.1016/j.medin.2018.06.010

3. Beltrán Salazar OA. La unidad de cuidado intensivo, un lugar para quedarse solo. Invest. educ. enferm. 2009;27(1):34-45.

4. Velasco Bueno JM, Alonso-Ovies A, Heras La Calle G, Zaforteza Lallemand C. Principales demandas informativas de los familiares de pacientes ingresados en Unidades de Cuidados Intensivos. Med Intensiva. 2018;42(6):337-45. https://doi.org/10.1016/j.medin.2017.09.007

5. Boada Quijano LC, Guáqueta Parada SR. Elementos para abordar la necesidad de información de las familias en una unidad de cuidados intensivos. Investig Enferm Imagen y Desarr. 2019;21(2). https://doi.org/10.11144/J averiana.ie21-2.eani

6. Achury Saldaña DM, Pinilla Alarcón M, Alvarado Romero H. Aspectos que facilitan o interfieren en el proceso comunicativo entre el profesional de enfermería y el paciente en estado crítico. Invest. educ. enferm. 2015;33(1):102-11. 
7. Zaforteza Lallemand C, García Mozo A, Amorós Cerdá SM, Pérez Juan E, Maqueda Palau M, Delgado Mesquida $\mathrm{J}$. Elementos facilitadores y limitadores del cambio en la atención a los familiares del paciente crítico. Enfermería Intensiva. 2012;23(3):121-31. https://doi.org/10.1016/j.enfi.2011.12.006

8. Sánchez-Vallejo A, Fernández D, Pérez-Gutiérrez A, Fernández-Fernández M. Análisis de las necesidades de la familia del paciente crítico y la opinión de los profesionales de la unidad de cuidados intensivos. Med Intensiva. 2016;40(9):527-40. https://doi.org/10.1016/j.medin.2016.03.005

9. Santana Cabrera L, Yañez Quintana B, Martín Alonso J, Ramírez Montesdeoca F, Roger Marrero I, Pérez de la Blanca AS. Actividades de comunicación del personal de enfermería con los familiares de pacientes ingresados en una unidad de cuidados intensivos. Enfermería Clínica. 2009;19(6):335-9. https://doi.org/10.1016/j.enfcli.2009.0 2.007

10. Pardavila Belio MI, Vivar CG. Necesidades de la familia en las unidades de cuidados intensivos: revisión de la literatura. Enfermería Intensiva. 2012;23(2):51-67. https://doi.org/10.1016/j.enfi.2011.06.001

11. Ballesteros Pinzón GA. Comunicación entre la enfermera y el familiar de la persona hospitalizada en la unidad de cuidados intensivos [tesis en Internet]. Bogotá: Universidad Nacional de Colombia; 2014 [citado 11 Sept 2020]. Disponible en: http://www.bdigital.unal.edu.co/47111/1/91506989.2014.pdf

12. Cotán A. El método etnográfico como construcción de conocimiento: un análisis descriptivo sobre su uso y conceptualización en ciencias sociales. Márgenes, Revista de Educación de la Universidad de Málaga. 2020;1(1), 83-103. http://dx.doi.org/10.24310/mgnmar.v1i1.7241

13. Bardin L. Análisis de contenido. $4^{\mathrm{a}}$ ed. Lisboa: Ediciones 70; 2010.

14. Polit D, Hungler B. Diseños de muestreo. En: Investigación científica en ciencias de la salud. $6^{a}$ ed. Ciudad de México: McGraw-Hill Interamericana; 2000.

15. Soto Lesmes VI, Durán de Villalobos MM. El trabajo de campo: clave en la investigación cualitativa. Aquichan. 2010;10(3):253-66.

16. Andréu Abela J. Las técnicas de análisis de contenido: una revisión actualizada. Sevilla: Fundación Centro de Estudios Andaluces.

17. Manzini JL. Declaración de Helsinki: principios éticos para la investigación médica sobre sujetos humanos. Acta bioeth. [Internet]. 2000; 6(2):321-334. http://www.scielo.cl/scielo.php?script=sci_arttext\&pid=S1726-569X 2000000200010\&lng=es. http://dx.doi.org/10.4067/S1726-569X2000000200010

18. Moscoso Loaiza LF., Díaz Heredia, LP. Aspectos éticos en la investigación cualitativa con niños. Rev Lat de Bioét. 2017;18(34-1), 51-67. https://doi.org/10.18359/rlbi.2955

19. García Ortega D, García Graus N, Martínez Martín ML. La relación terapéutica con la familia del paciente crítico. Metas Enferm. 2016 Mar;19(2):49-59.

20. Cerón Polanco YM. Aspectos presentes en la comunicación de la enfermera, la familia y la institución en la unidad de cuidado intensivo [tesis en Internet]. Bogotá: Pontificia Universidad Javeriana; 2008 [citado 10 Sept 2020]. Disponible en: https://www.javeriana.edu.co/biblos/tesis/enfermeria/tesis52.pdf

21. Marquez Herrera M, Carrillo González GM. La experiencia del familiar de la unidad de cuidados intensivos en Bucaramanga: un estudio fenomenológico. Arch Med (Manizales). 2015;15(1):95-106. https://doi.org/10.305 54/archmed.15.1.720.2015

22. Rincón Cepeda CM. La comunicación terapéutica entre la enfermera y el paciente como habilidad de cuidado humanizado en la UCI: una revisión de tema [tesis en Internet]. Bogotá: Universidad de Ciencias Aplicadas y Ambientales UDCA; 2017 [citado 10 Sept 2020]. Disponible en: https://repository.udca.edu.co/bitstream/11 158/641/1/COMUNICACION TERAPEUTICA ENTRE ENFERMERA Y PACIENTE. HABILID.pdf

23. Arica Cieza VJ. El cuidado de enfermería al adulto mayor hospitalizado: complementariedad, calor humano e interculturalidad [tesis]. Chiclayo: Universidad Católica Santo Toribio de Mogrovejo; 2017.

24. Noreña Peña AL, Cibanal Juan L, Alcaraz Moreno N. La interacción comunicativa en el cuidado de la salud. Rev Esp Com Sal. 2010;1(2):113-29.

25. Cánovas Tomás MÁ. La relación de ayuda en enfermería: Una lectura antropológica sobre la competencia relacional en el ejercicio de la profesión [tesis]. Murcia: Universidad de Murcia; 2008. 
26. Ramírez P, Müggenburg C. Relaciones personales entre la enfermera y el paciente. Enfermería Universitaria. 2015;12(3):134-43. https://doi.org/10.1016/j.reu.2015.07.004

27. Nava Galán MG, Zamora Ruíz P. Percepción del trato digno de los familiares de los enfermos neurológicos en el Instituto Nacional de Neurología y Neurocirugía. Enf Neurol (Mex). 2010;9(1):32-8.

28. Campo Martínez MC, Cotrina Gamboa MJ. Relación de ayuda al familiar del paciente en situación crítica. Enferm. glob. 2011;10(24). http://dx.doi.org/10.4321/S1695-61412011000400009

\section{Notas}

* Artículo de investigación

1 La "E" corresponde al número de la entrevista, la "p" corresponde a la página y el último número es el renglón donde se ubica la cita.

\section{Licencia Creative Commons CC BY 4.0}

Cómo citar este artículo: Díaz Heredia LP, Ballesteros Pinzón GA. Comunicación entre la enfermera y el familiar: una relación entre seres humanos honesta, directa y real. Investig Enferm Imagen Desarr. 2021;23. https://doi.org/10.11144/Javeriana.ie23.cefr. 